\title{
Detector for coherent synchrotron radiation measurements from separate electron bunches in a millimeter wavelength region
}

\author{
G. Naumenko ${ }^{\mathrm{a}, *}$, A. Potylitsyn ${ }^{\mathrm{b}}$, G. Kube ${ }^{\mathrm{c}}$, O. Grimm $^{\mathrm{d}}$, V. Cha ${ }^{\mathrm{b}}$, Yu. Popov $^{\mathrm{b}}$ \\ a Nuclear Physics Intitute at Tomsk Polytechnic university, Lenina Avenue 2a, 634050, Tomsk, Russia \\ b Tomsk Polytechnic university, Lenina Avenue 2, 634050, Tomsk, Russia \\ ${ }^{\mathrm{c}}$ DESY, Hamburg, Germany \\ ${ }^{\mathrm{d}}$ University of Hamburg, Luruper Chaussee 149, 22761 Hamburg, Germany
}

\section{A R T I C L E I N F O}

Available online 3 January 2009

Keywords:

Detector

Millimeter wavelength

Synchrotron radiation

\begin{abstract}
A B S T R A C T
Room-temperature detector, manufactured by the company "SPINOR" (Tomsk Russia) was tested for the investigations of the coherent synchrotron radiation characteristics at the bent electron beam of FLASH (Hamburg) in the wavelength region $0.5-3 \mathrm{~mm}$. Detector is based on a low-threshold diode and a broadband antenna with a built-in preamplifier. The detector characteristics allowed us to measure a radiation pulse from separate electron bunches from electron train. The measured coherent synchrotron radiation spectral characteristics in comparison with ones, measured using the cryogenic bolometer show a good homogeneity of the spectral sensitivity of our detector for wavelengths longer than $1.5 \mathrm{~mm}$. The comparison of temporal characteristics with the well-established pyro-electric detectors shows a response time around $1.5 \mathrm{~ns}$. The measured detector sensitivity was about $100 \mathrm{MV} / \mathrm{J}$.
\end{abstract}

(c) 2008 Elsevier B.V. All rights reserved.

\section{Design of detector DPU-1}

The room-temperature detector DPU-1 is intended for measurement of the radiation in a millimeter wavelength region. Detector was manufactured by the company "SPINOR" (Tomsk, Russia). Main elements of the detector are the low-threshold diode, broadband antenna and fast preamplifier.

Standard power supply of $12 \mathrm{~V}$ is provided in a complete set with detector. The main applicability of detector is a measurement of different types of coherent radiation from a single submillimeter length bunch of charged particles for a purpose of beam diagnostics. The detector has a sensitive area of $4 \mathrm{~mm}^{2}$ and registers radiation without any horn.

\section{Detector response}

Test was performed using the coherent synchrotron radiation (CSR) beam, which is generated from the edge of bending magnet placed downstream to the first bunch compressor in line of injector of FLASH (Hamburg, Germany).

CSR beam is extracted from accelerator vacuum chamber through the vacuum window of the beamline of CF63-type with the outcoupling area of $60 \mathrm{~mm}$ wide and $8 \mathrm{~mm}$ high, converted to

\footnotetext{
*Corresponding author. Tel.: +73822 423994; fax: +73822423934

E-mail address: naumenko@npi.tpu.ru (G. Naumenko).
}

the parallel beam and transported by the optical tract to the TOSYLAB laboratory [1].

Main electron beam characteristics are shown in Table 1.

Fig. 1 shows the sample of detector response on the CSR from a single bunch.

The pulse consists of two components. The main component has the amplitude $20-30 \mathrm{mV}$ and the width $1.5 \mathrm{~ns}$ (FWHM). The area under the peak is $\approx 35 \mathrm{mV}$ ns. Besides the main peak there is slow component with an average amplitude $\approx 2.5 \mathrm{mV}$, which reaches the duration $\approx 150 \mathrm{~ns}$. The area under the shape is $\approx 380 \mathrm{mV}$ ns. Both are a response of the radiation and are proportional to the radiation energy. As the temporal window of analog-digital converter (ADC) is about $8 \mathrm{~ns}$, we can provide the measurement using the main peak component with the good accuracy. Nevertheless, the shape limits the temporal resolving possibility if an incoming bunches period is less than $150 \mathrm{~ns}$.

As shown in Fig. 1, the peak/noise ratio for observed signal is $\eta_{\mathrm{DPU}-1} \approx 40$. This is the important criterion of detector sensitivity. For comparison, the value of the peak/noise ratio for pyro-electric detector DTGS (see Ref. [2]) is $\eta_{\text {DTGS }} \approx 3.3$.

Usually the detector responsivity criterion is defined as the relation of a detector response peak voltage to a radiation pulse energy $E_{\mathrm{r}}$ (if the response duration is much larger than radiation pulse duration). However, such criterion does not define unambiguously the detector responsivity characteristics, because it strongly depends on a preamplifier and additional amplifier properties. In this case, the more correct criterion of detector sensitivity is the relation $\chi=\eta / E_{\mathrm{r}}$. The inverse value of $\chi$ is the 
Table 1

Main electron beam characteristics.

Single-bunch charge: $0.6-0.9 \mathrm{nCb}\left(\approx 4 \times 10^{9} \mathrm{e}^{-}\right)$

Single-bunch length: $100 \mathrm{fs}$ ( $<300 \mathrm{um}$ )

Radiation energy from single bunch $\approx 50-100 \mathrm{n} J$

Lorenz-factor: 300

A train consists of 1-100 single bunches

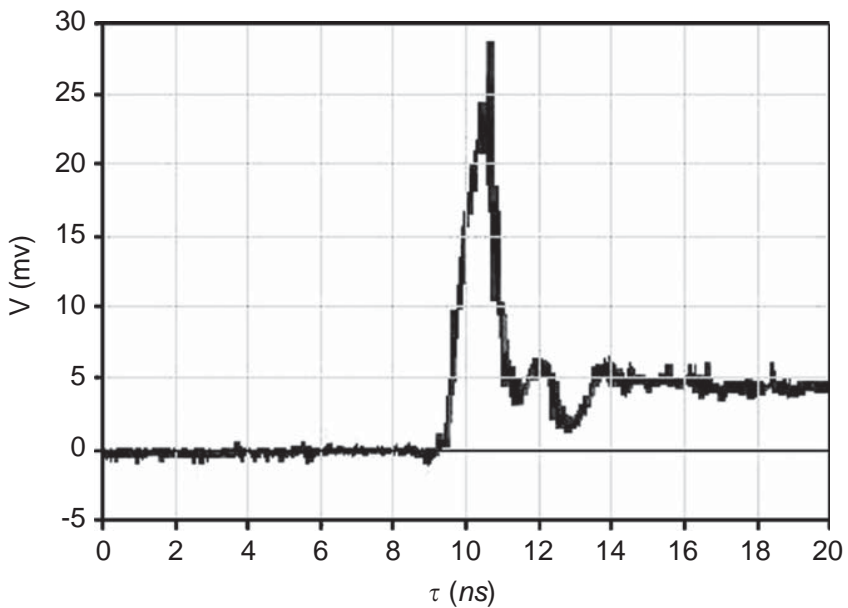

Fig. 1. Detector DPU-1 response on radiation from single bunch with scale $2 \mathrm{~ns}$ per division.

radiation energy, which provides the unity enhancement of the detector response over the noise.

For detector DPU-1, the value of this criterion is equal $\chi \approx 40 /$ $80 \mathrm{~nJ}=0.5 \times 10^{9} \mathrm{~J}^{-1}$, and for detector DTGS $\chi \approx 3.3 / 80 \mathrm{~nJ}=0.4 \times 10^{8}$ $\mathrm{J}^{-1}$

\section{Spectral characteristics}

The spectral measurements using the pair of DPU-1 detectors were performed using Martin-Puplett interferometer of TOSYLAB in DESY [3]. Not any additional amplifier was used for spectral measurements. For comparison, the similar measurements were done using the pair of pyro-electric detectors DTGS for the same electron beam conditions. For the spectra reconstruction from interferograms was used the Martin-Puplett interferometer builtin program system and the similar program system, based on the theoretical description of the spectra reconstruction process, described in Ref. [3].

As the DTGS detector does not separate two sequential bunches, the measurements for detector properties comparison were performed for beam operation in the single-bunch mode. In Figs. 2 and 3 difference interferograms obtained using the pair of DTGS detectors and the pair of DPU-1 are shown.

The radiation spectra reconstructed from these interferograms are presented in Fig. 4. The suppression of the hard part in the spectrum obtained using detector DPU-1 (Fig. 4) probably is caused by the detector spectral efficiency, which is limited by the frequency $\approx 0.3 \mathrm{THz}$. The mentioned gap in spectra from DTGS, we explain by the abnormal absorption of SR in radiation path.

Taking into account that the spectral efficiency of a cryogenic bolometer is almost constant for a wide spectral range (much more than region of spectral sensitivity of DPU-1), we may estimate the DPU-1 spectral efficiency as a ratio of spectra,

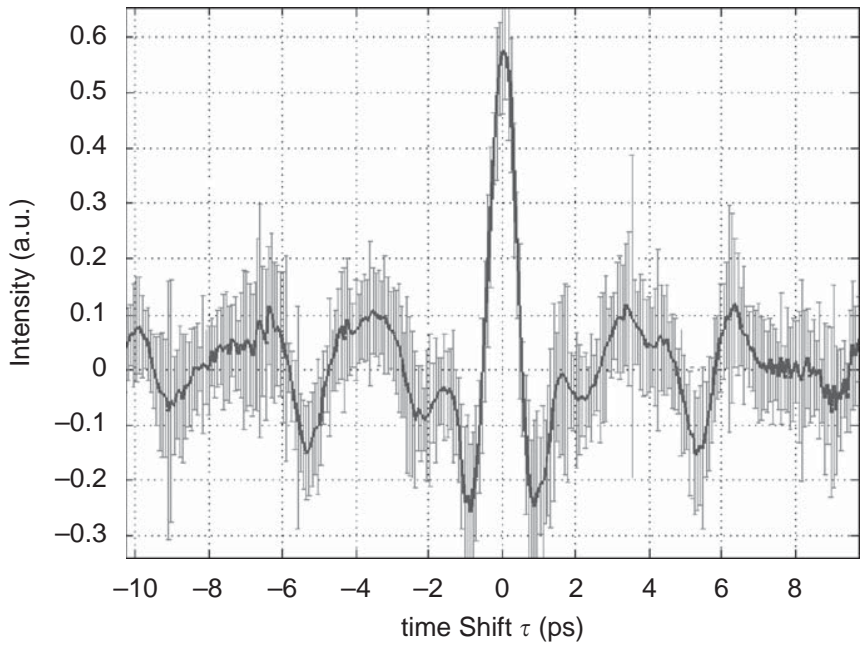

Fig. 2. The difference interferogram using pair of DTGS detectors.

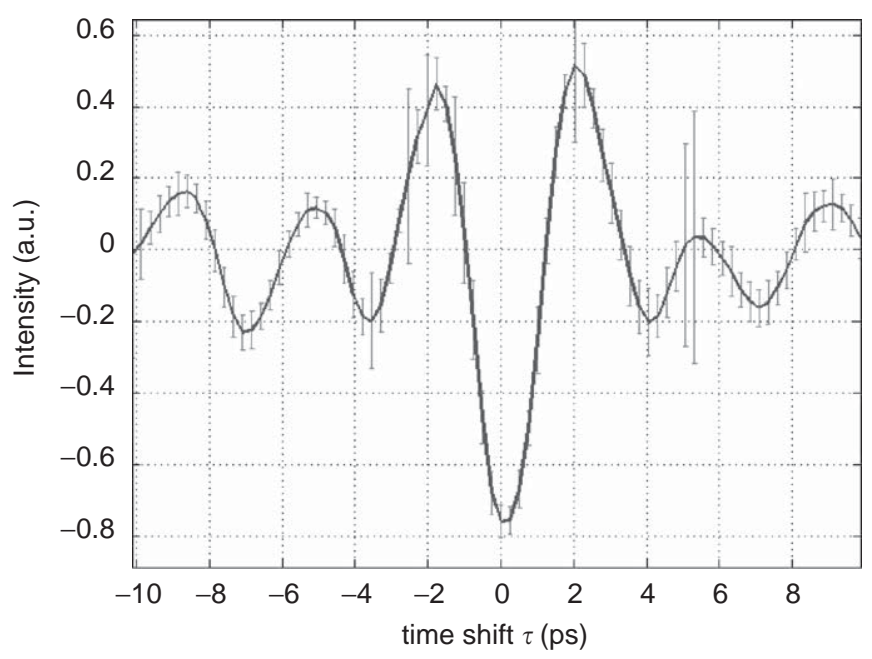

Fig. 3. The difference interferogram using pair of DPU-1 detectors.

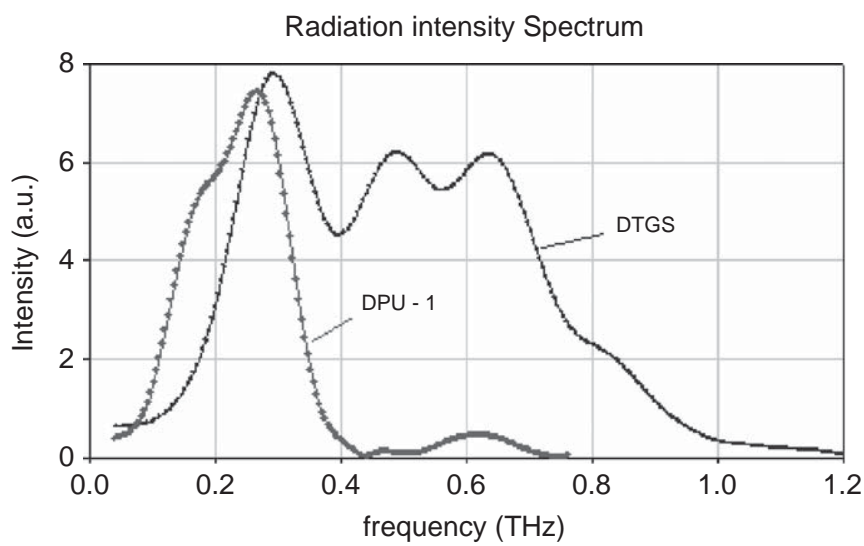

Fig. 4. The CSR reconstructed spectrum from pair of DPU-1 detectors and pair of DTGS detectors.

measured with DPU-1 and cryogenic bolometer, using the same spectra recovering the procedure (see Fig. 5).

Fig. 5 shows the radiation efficiency for radiation wavelength $>1.5 \mathrm{~mm}$ is almost constant. The high peak/noise ratio, which is 


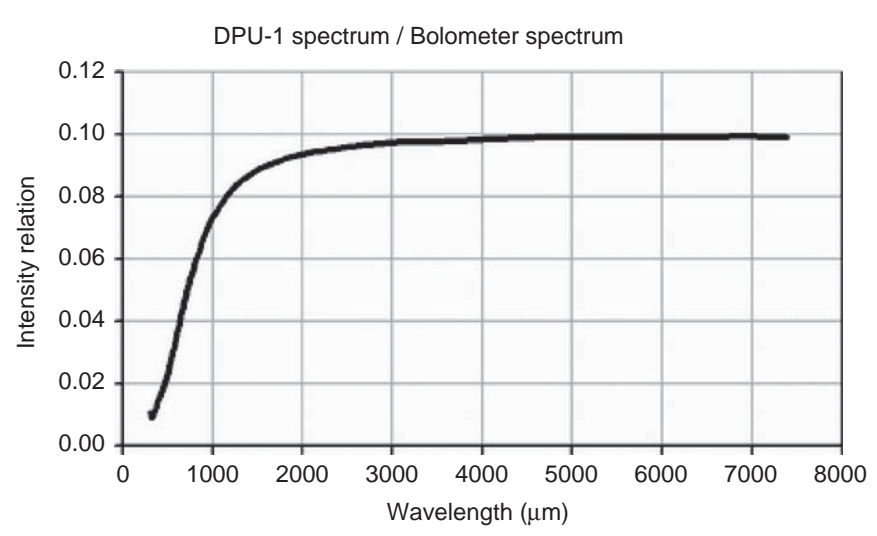

Fig. 5. The ratio of the spectra measured with DPU-1 and cryogenic bolometer.

peculiar for the DPU-1 detector allows reconstructing a spectrum from an interferogram measured by a single detector. Such reconstruction is impossible for the spectra from DTGS detector, measured in single-bunch mode due to high noise contribution.

\section{Absolute calibration}

For estimation of DPU-1 detector absolute sensitivity, we used the absolute calibration of DTGS detector, presented in Ref. [4], where the average responsivity is $S_{\mathrm{DTGS}} \approx 35 \mathrm{MV} / \mathrm{J}$. The DTGS detector passport data allows us to assume that this value is valid for radiation frequency down to $0.25 \mathrm{THz}$. For estimation the DPU1 detector sensitivity, we used the absolute difference of interferograms $\Delta V$ measured using the pair of detectors DPU-1 and the similar difference, measured using the pair of detectors DTGS in a single-bunch mode (see Fig. 6).

The measured difference is $42 \mathrm{mV}$ near the main maximum for DPU-1 and $13.6 \mathrm{mV}$ for DTGS. So the absolute sensitivity of the DPU-1 detector may be defined as

$S_{\text {DPU- } 1}=\frac{\Delta V_{\text {DPU-1 }}}{\Delta V_{\text {DTGS }}} S_{\text {DTGS }}=\frac{42}{13.6} 35 \mathrm{MV} / \mathrm{J}=108 \mathrm{MV} / \mathrm{J}$

\section{Summary}

The detector DPU-1 based on the low-threshold diode and broadband antenna for radiation measurement in a millimeter

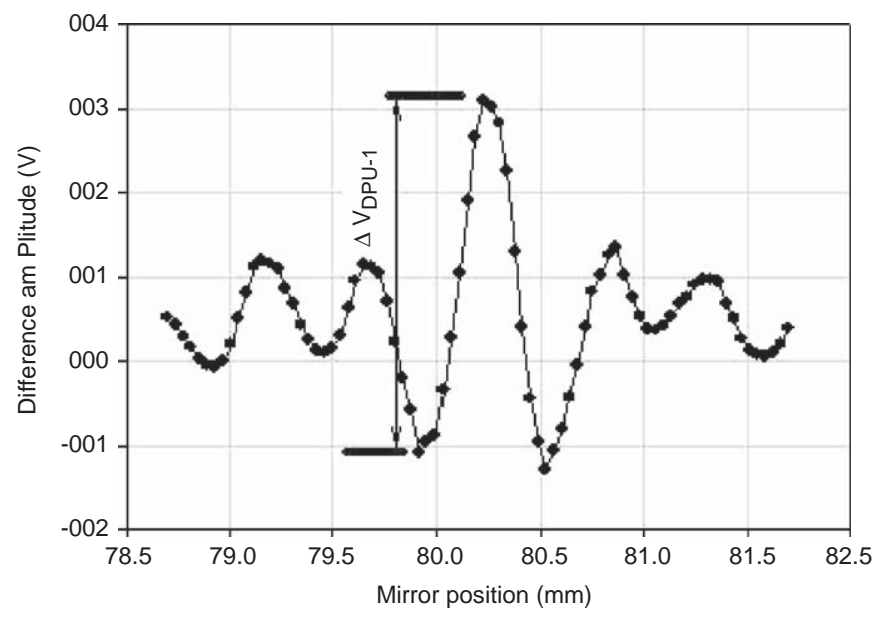

Fig. 6. Direct difference of interferograms, measured using pair of detectors DPU1. $\Delta V_{\mathrm{DPU}-1}=42 \mathrm{mV}$.

wavelength region was tested at the coherent synchrotron radiation beam from the bunched electron beam at FLASH.

The temporal detector characteristics allow investigating radiation characteristics from a separate electron bunch with time interval $150 \mathrm{~ns}$. The detector pulse duration was about $1.5 \mathrm{~ns}$ (FWHM). The detector response demonstrates a good peak-tonoise ratio, which is approximately 40 in contrast with DTGS detector (around 3.3) with respect to the single-bunch measurements. The spectral sensitivity of detector is approximately constant for radiation frequency less than $0.3 \mathrm{THz}$ and it decreases for high-frequency $(>0.3 \mathrm{THz})$ radiation. The absolute detector sensitivity for the radiation frequency less than $0.3 \mathrm{THz}$ is approximately $100 \mathrm{MV} / \mathrm{J}$. The similar simple and reliable roomtemperature detector may be used for the FEL beams diagnostics technique.

\section{References}

[1] O. Grimm, et al., The Synchrotron radiation beamline at TTF2, in: Proceedings of the EPAC 2004, Lucerne, 5-9 July 2004, p. 2586.

[2] Oliver Grimm, Operation principles of pyroelectric detectors, DESY, MPY, 10 June 2004.

[3] Lars Froehlich, Bunch length measurements using a Martin-Puplett interferometer at the VUV-FEL, DESY-THESIS 2005-011, FEL-THESIS 2005-02.

[4] E. Chiadroni, H. Delsim-Hashemi, L. Froehlich, O. Grimm, Report on the visit at FELIX, FOM Rijnhuizen, DESY preprint, 1-3 March 2005. 